\title{
Long Survival after Gastrointestinal Bleeding in a New-Onset Heyde's Syndrome Patient Treated with Multiple Endoscopic Hemostatic Procedures and Repeated Transfusions after Aortic Valve Replacement
}

\author{
Genso Notoya $^{a}$ Ryota Niikura ${ }^{a}$ b Atsuo Yamada $^{a}$ Masanori Ochi ${ }^{a}$ \\ Takashi Kawai $^{\text {b }}$ Kazuhiko Koike $^{a}$ \\ aDepartment of Gastroenterology, Graduate School of Medicine, The University of Tokyo, \\ Tokyo, Japan; ${ }^{b}$ Gastroenterological Endoscopy, Tokyo Medical University, Tokyo, Japan
}

Keywords

Heyde's syndrome · Gastrointestinal bleeding · Endoscopic hemostasis · Transfusion · Long survival

\begin{abstract}
Heyde's syndrome, which is caused by aortic stenosis and subsequent acquired von Willebrand factor deficiency, is a gastrointestinal bleeding disease. Gastrointestinal bleeding develops in patients with Heyde's syndrome, which may have a different prognosis from general gastrointestinal bleeding; thus, it is important to understand the clinical course. We report a 76-year-old Japanese female who underwent aortic mechanical valve replacement 1 year ago and presented with recurrent gastrointestinal bleeding in angiodysplasia of the sigmoid colon. Endoscopic interventions achieved hemostasis. However, 6 rebleeding events occurred due to a sigmoid colon ulcer and gastric and jejunal angiodysplasia 7 years after first hemostasis. The patient underwent multiple endoscopic hemostatic procedures (upper, lower, and balloon-assisted endoscopy) and repeated transfusions (total of 394 units of red blood cells). The intensive treatment contributed to the survival time of 10 years. In addition, we performed a literature review of the prognosis of patients with Heyde's syndrome.
\end{abstract}

(C) 2021 The Author(s).

Published by S. Karger AG, Basel 
Notoya et al.: A Case of Heyde's Syndrome with Long Survival

\section{Introduction}

Gastrointestinal bleeding is a common clinical condition requiring hospitalization and blood transfusion, particularly in patients with severe systemic comorbidities, including cardiovascular diseases such as Heyde's syndrome [1,2]. The pathogenesis of Heyde's syndrome is characterized by gastrointestinal bleeding caused by arteriovenous malformation and acquired von Willebrand factor (vWF) deficiency resulting from mechanical destruction of vWF multimers as they pass through the narrowed aortic valve. Gastrointestinal bleeding lesions develop in patients with Heyde's syndrome, which may have a different prognosis from general gastrointestinal bleeding; thus, it is important to understand the clinical course.

We report a case of Heyde's syndrome characterized by chronic persistent gastrointestinal bleeding that was treated with multiple endoscopic hemostatic procedures and transfusions, resulting in a survival time of approximately 10 years. We also performed a literature review of the prognosis of patients with Heyde's syndrome.

\section{Case Report/Case Presentation}

A 76-year-old woman underwent mitral valve replacement and tricuspid valve in 1989. The patient also underwent aortic valve replacement in 2009 due to advanced aortic stenosis and regurgitation.

A large-volume painless tarry stool occurred in 2010, accompanied by hemorrhagic shock. A complete blood count revealed a hemoglobin level of $11.0 \mathrm{~g} / \mathrm{dL}$. The patient required a transfusion to recover her general condition. Early colonoscopy identified the stigmata of a recent hemorrhage for angiodysplasia of the sigmoid colon, and she underwent endoscopic coagulation (Fig. 1A).

Between 2010 and 2016, 6 gastrointestinal bleeding events occurred in a sigmoid colon ulcer, sigmoid and gastric angiodysplasia, and a descending colon polyp, which required the transfusion of 10 units of blood. Endoscopic therapies, including clipping and argon plasma coagulation (APC), successfully treated these gastrointestinal bleeding (Fig. 1B, C).

In 2016, anemia occurred after gastric hyperplastic polyp bleeding due to a Helicobacter pylori (H. pylori) infection and required 16 units of blood. Endoscopic mucosal resection was performed, and $H$. pylori was eradicated. However, the anemia progressed again in 2017. Capsule endoscopy was performed and revealed jejunal intestinal angiodysplasia. APC was performed using balloon-assisted endoscopy (Fig. 1D). A total of 360 units of blood were transfused between 2017 and 2020 to treat the slowly progressing anemia (Fig. 2).

The patient had a max aortic jet velocity $\left(A V_{\max }\right)$ of $3.96 \mathrm{~m} / \mathrm{s}$ in 2008 before the aortic valve replacement, which improved to $3.04 \mathrm{~m} / \mathrm{s}$ after surgery in 2009 . The $A V_{\text {max }}$ increased to $3.95 \mathrm{~m} / \mathrm{s}$ in 2010 when gastrointestinal bleeding occurred, and increased to $4.74 \mathrm{~m} / \mathrm{s}$ in 2012 (Fig. 3). The vWF activity decreased from 113\% in 2017 to $100 \%$ in 2019 . The vWF multimer analysis was normal in 2020; her vascular endothelial growth factor level was 865 $\mathrm{pg} / \mathrm{mL}$ (normal range: $62-704 \mathrm{pg} / \mathrm{mL}$ ).

Hematemesis developed in October 2020. A complete blood count revealed progression of anemia (hemoglobin level $=6.8 \mathrm{~g} / \mathrm{dL}$ ), which required 24 units of blood. Upper endoscopy revealed multiple gastric angiodysplasia lesions, and hemostasis was performed twice using APC (Fig. 1E, F). Although hemostasis was achieved, the patient died of acute exacerbation from chronic heart failure in November 2020, 10 years after the initial gastrointestinal bleeding event.

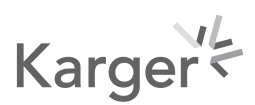




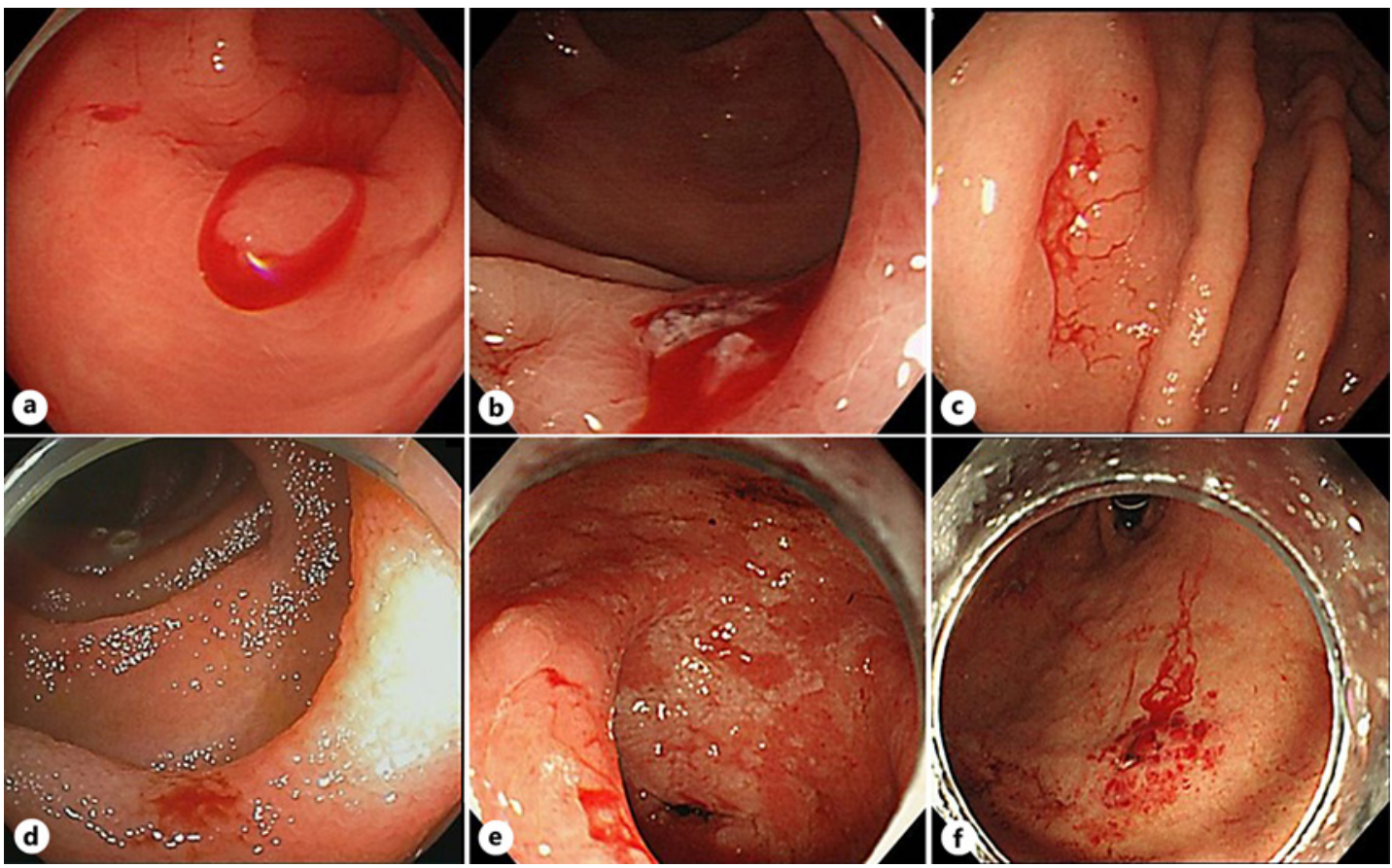

Fig. 1. Gastrointestinal bleeding events. Active bleeding in angiodysplasia of the sigmoid colon in 2010 (A), 2013 (B), and 2016 (C). D Jejunal angiodysplasia in 2017. E, F Active bleeding in gastric angiodysplasia in 2020.

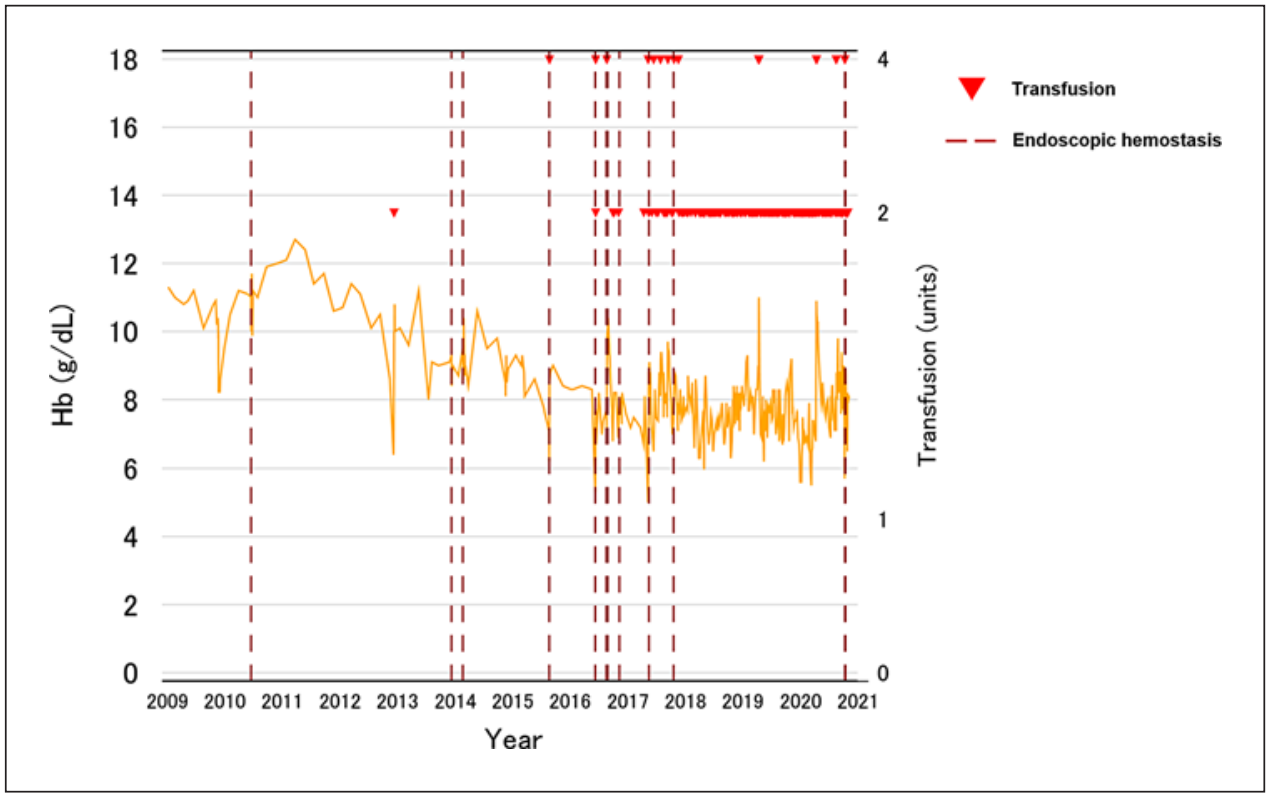

Fig. 2. Clinical course of the patient. Yellow line shows the hemoglobin level.

\section{Discussion/Conclusion}

We performed multiple endoscopic hemostatic procedures in a patient with new-onset Heyde's syndrome after aortic valve replacement. The patient survived for approximately 10 

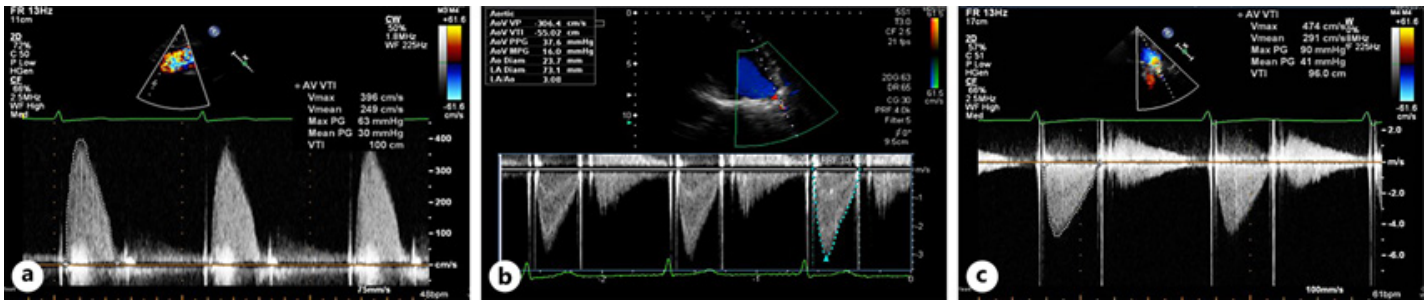

Fig. 3. Changes on echocardiography. A Preoperative transthoracic echocardiography shows severe aortic stenosis $\left(\mathrm{AV}_{\max }=3.96 \mathrm{~m} / \mathrm{s}\right)$. B Postoperative transthoracic echocardiography reveals improved aortic stenosis $\left(\mathrm{AV}_{\max }\right.$ $=3.06 \mathrm{~m} / \mathrm{s}) \cdot$ C Transthoracic echocardiography shows exacerbation of aortic stenosis $\left(\mathrm{AV}_{\max }=4.74 \mathrm{~m} / \mathrm{s}\right)$.

years after the first gastrointestinal bleeding event. To our knowledge, this is the first report of a long survival time in a new-onset Heyde's syndrome patient after aortic valve replacement.

In 1958, Heyde [1] reported an association between gastrointestinal bleeding and aortic stenosis. The criteria for Heyde's syndrome changed to aortic stenosis with intestinal angiodysplasia and acquired vWF syndrome; our patient met those criteria. Importantly, the time of onset after aortic valve replacement was approximately 1 year, consistent with a previous report [3].

Table 1 shows the previous reports detailing the clinical characteristics and prognoses of patients with Heyde's syndrome. Chukwudum et al. [4] reported a 61-year-old man with upper gastrointestinal bleeding who survived for 36 months. Hudzik et al. [5] reported an 82-year-old man with a bleeding colon who survived 12 months. Our patient survived longer than those in all previous reports [3, 4, 6-18]. However, our patient developed Heyde's syndrome after cardiac surgery, and we could not perform further cardiac interventions. The treatments for Heyde's syndrome include managing cardiac function and gastrointestinal bleeding. Multiple endoscopic hemostatic procedures may contribute to a good response in a patient with Heyde's syndrome. Another potential reason for the long survival of our patient may be the repeated transfusions. Heyde's syndrome is an acquired vWF disease. A severely narrowed aortic valve causes high shear stress and stretching, which impairs function [1]. Multiple transfusions contribute to the recovery of vWF multimer function and activity [19]. In conclusion, multiple endoscopic hemostatic procedures and transfusions may be effective to prolong the survival time of gastrointestinal bleeding patients with Heyde's syndrome.

\section{Statement of Ethics}

This study protocol was reviewed and approved by the Institutional Review Board of the University of Tokyo Hospital, Approval No. [2058-2]. Written informed consent was obtained from the patient for publication of this case report and any accompanying images.

\section{Conflict of Interest Statement}

The authors have no conflicts of interest to declare.

\section{Funding Sources}

This study was supported by KAKENHI Grants-in-Aid for Scientific Research (grant number 20K08375) (R.N.).

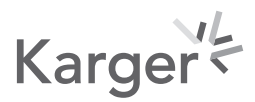




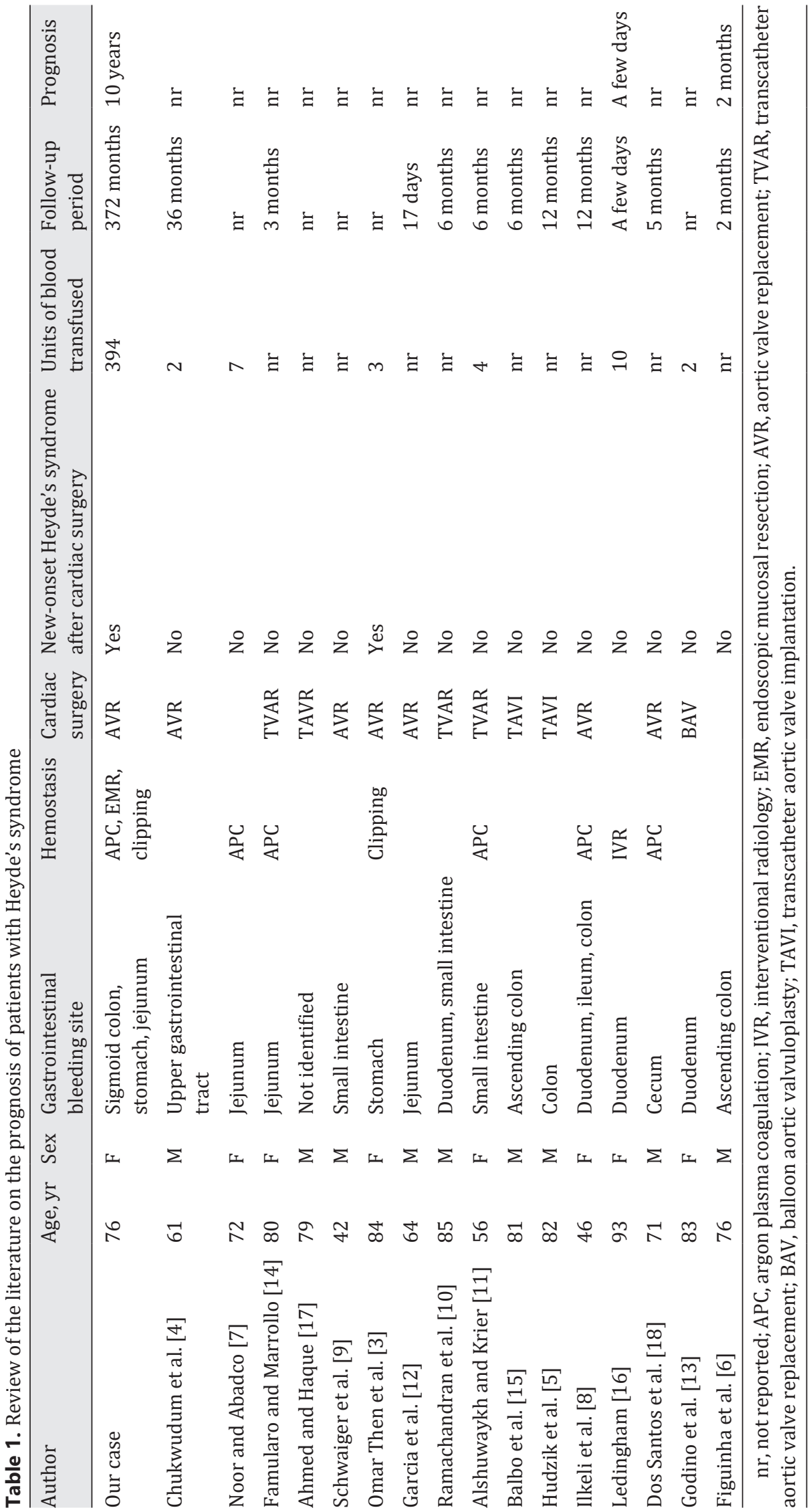


Notoya et al.: A Case of Heyde's Syndrome with Long Survival

\section{Author Contributions}

G. Notoya and R. Niikura contributed to literature review and manuscript writing. M. Ochi performed the management of the patient. A. Yamada contributed to supervision. T. Kawai and K. Koike contributed to final review of the manuscript. The final version of the manuscript was read and approved by all authors.

\section{Data Availability Statement}

The Tokyo University IRB does not allow access to the study data on the basis of ethical grounds.

\section{References}

1 Heyde EC. Gastrointestinal bleeding in aortic stenosis. N Engl J Med. 1958;259(4):196.

2 Vincentelli A, Susen S, Le Tourneau T, Six I, Fabre O, Juthier F, et al. Acquired von Willebrand syndrome in aortic stenosis. N Engl J Med. 2003 Jul 24;349(4):343-9.

3 Omar Then E, Catalano C, Sunkara T, Gaduputi V. New onset Heyde's syndrome presenting after total aortic valve replacement. Oxf Med Case Reports. 2019 Feb 16;2019(2):omy134.

4 Chukwudum CA, Vera S, Sharma M, Varon J, Surani S. Heyde Syndrome: a case report and literature review. Cureus. 2020 Apr 30;12(4):e7896.

5 Hudzik B, Wilczek K, Gasior M. Heyde syndrome: gastrointestinal bleeding and aortic stenosis. CMAJ. 2016 Feb 2;188(2):135-8.

6 Figuinha FC, Spina GS, Tarasoutchi F. Heyde's syndrome: case report and literature review. Arq Bras Cardiol. 2011 Mar;96(3):e42-5.

7 Noor A, Abadco D. Heyde syndrome complicated by a Dieulafoy lesion. Ochsner J. 2020 Fall;20(3):326-30.

8 Ilkeli E, Albeyoglu S, Ciloglu U, Sabri D. Heyde's syndrome. Asian Cardiovasc Thorac Ann. 2014 Jun;22(5): 592-4.

9 Schwaiger JP, Ludwiczek O, Graziadei I, Grander W. A vicious circle: Heyde syndrome in mild aortic stenosis. CASE. 2019 May 31;3(4):171-6.

10 Ramachandran R, Uqdah H, Jani N. A case of recurrent obscure gastrointestinal bleeding: Heyde's syndrome - case report and review. J Community Hosp Intern Med Perspect. 2018 Jun 12;8(3):127-9.

11 Alshuwaykh 0, Krier MJ. A case of Heyde syndrome with resolution of gastrointestinal bleeding two weeks after aortic valve replacement. Am J Case Rep. 2018 Aug 7;19:924-6.

12 Garcia LR, Garzesi AM, Tripoli G, Campos NLKL, Martins AS, Felicio ML. Heyde syndrome treated by conventional aortic valve replacement. Braz J Cardiovasc Surg. 2019 Dec;34(5):630-2.

13 Godino C, Pavon AG, Mangieri A, Margonato A. Aortic valvuloplasty as bridging for TAVI in high-risk patients with Heyde's syndrome: a case report. Case Rep Med. 2012;2012:946764.

14 Famularo G, Marrollo M. Of aortic valve and bleeding: Heyde's syndrome. Am J Emerg Med. 2020 Nov;38(11): 2493.e1-2.

15 Balbo CP, Seabra LP, Galoro VG, Caputi G, Palma JH, Buffolo Ê. Heyde’s syndrome and transcatheter aortic valve implantation. Arq Bras Cardiol. 2017 Apr;108(4):378-80.

16 Ledingham D. Heyde's syndrome: exploring the link between aortic stenosis and an acquired bleeding disorder. BMJ Case Rep. 2013 Apr 18;2013:2013.

17 Ahmed T, Haque R. Heyde's syndrome complicating management in a patient with high bleeding and thrombotic risks. Cureus. 2020 May 25;12(5):e8280.

18 Dos Santos VM, Dos Santos LA, Modesto AA, Amui MO. Heyde syndrome in a 71-year-old man who underwent chest radiotherapy at young age. An Sist Sanit Navar. 2013 Sep 6;36(2):339-45.

19 Kuroda G, Takase H, Toriyama T, Okada T, Hagikura A, Takagaki K. A case of Heyde syndrome. Jpn Heart. 2011; 44:37-41. 